\title{
The Future of High-Energy Astrophysical Neutrino Flavor Measurements
}

\section{Ningqiang Song, ${ }^{a, b, c, *}$ Shirley Weishi Li, ${ }^{d, e}$ Carlos A. Argüelles, ${ }^{f}$ Mauricio Bustamante $^{g}$ and Aaron C. Vincent ${ }^{a, b, c}$}

${ }^{a}$ Department of Physics, Engineering Physics and Astronomy, Queen's University, Kingston ON K7L 3N6, Canada

${ }^{b}$ Arthur B. McDonald Canadian Astroparticle Physics Research Institute, Kingston ON K7L 3N6, Canada

${ }^{c}$ Perimeter Institute for Theoretical Physics, Waterloo ON N2L 2Y5, Canada

${ }^{d}$ SLAC National Accelerator Laboratory, 2575 Sand Hill Road, Menlo Park, CA, 94025, USA

${ }^{e}$ Theoretical Physics Department, Fermilab, P.O. Box 500, Batavia, IL 60510, USA

${ }^{f}$ Department of Physics \& Laboratory for Particle Physics and Cosmology, Harvard University, Cambridge, MA 02138, USA

${ }^{g}$ Niels Bohr International Academy \& DARK, Niels Bohr Institute,University of Copenhagen, DK-2100 Copenhagen, Denmark

E-mail: ningqiang.song@queensu.ca, shirleyl@fnal.gov, carguelles@fas.harvard.edu, mbustamante@nbi.ku.dk, aaron.vincent@queensu.ca

The next generation of neutrino telescopes, including Baikal-GVD, KM3NeT, P-ONE, TAMBO, and IceCube-Gen2, will be able to determine the flavor of high-energy astrophysical neutrinos with $10 \%$ uncertainties. With the aid of future neutrino oscillation experiments - in particular JUNO, DUNE, and Hyper-Kamiokande - the regions of flavor composition at Earth that are allowed by neutrino oscillations will shrink by a factor of ten between 2020 and 2040. We critically examine the ability of future experiments and show how these improvements will help us pin down the source of high-energy astrophysical neutrinos and a sub-dominant neutrino production mechanism with and without unitarity assumed. As an illustration of beyond-the-Standard-Model physics, we also show that the future neutrino measurements will constrain the decay rate of heavy neutrinos to be below $2 \times 10^{-5} \mathrm{~m} / \mathrm{eV} / \mathrm{s}$ assuming they decay into invisible particles.

$37^{\text {th }}$ International Cosmic Ray Conference (ICRC 2021)

July 12th - 23rd, 2021

Online - Berlin, Germany

\footnotetext{
${ }^{*}$ Presenter
} 


\section{Introduction}

High energy astophysical neutrinos are produced in hadronic accelerators where high-energy protons and nuclei interact with surrounding matter and radiation. These neutrinos are detected in large-scale neutrino telescopes, which identify the energy spectrum, arrival directions, arrival times and in particular the flavor composition of high-energy neutrinos, i.e. the proportion of $v_{e}, v_{\mu}$ and $v_{\tau}$ in the neutrino flux. The information they carry offfers unprecedented insight into astrophyscics and fundamental physics, including the unidentified sources of high energy astrophysical neutrinos and the new physics in neutrino production, propagation and detection [1].

At present, however, our ability to identify the neutrino sources and new physics is hampered by two important yet surmountable limitations. On the statistical side, more observations are demanded to reconstruct the flavor of high-energy astrophysical neutrinos at Earth. On the systematical side, precise knowledge of neutrino mixing parameters is required to map the flavor composition at the source to that at Earth.

Fortunately, these limitations will be overcome in the next two decades, thanks to new terrestrial and astrophysical neutrino experiments that are planned or in construction. Oscillation experiments that use terrestrial neutrinos-JUNO [2], DUNE [3], Hyper-Kamiokande (HK) [4], and the IceCube-Upgrade [5] — will reduce the uncertainties in the mixing parameters and put the standard oscillation framework to test. Large-scale neutrino telescopes-Baikal-GVD [6], IceCubeGen2 [7], KM3NeT [8], P-ONE [9], and TAMBO [10]—will detect more high-energy astrophysical neutrinos and improve the measurement of their flavor composition.

In this work, we show realistic projections of how the determination of how the uncertainty in the predicted flavor composition at Earth of the isotropic flux of high-energy neutrinos and its measurement will evolve over the next two decades. Our main finding is that, by 2040, we will be able to precisely infer the flavor composition at the sources, including possibly identifying the contribution of multiple neutrino-production mechanisms, even if oscillations are non-unitary. Further, we illustrate the upcoming power of flavor measurements to probe BSM neutrino physics using neutrino decay.

\section{Future neutrino oscillation experiments}

In the standard oscillation scenario, the flavor and mass states are related via a unitary transformation, i.e.,

$$
\left|v_{\alpha}\right\rangle=\sum_{i=1}^{3} U_{\alpha i}^{*}\left|v_{i}\right\rangle
$$

where $\alpha=e, \mu, \tau$, and $U$ is the Pontecorvo-Maki-Nakagawa-Sakata (PMNS) lepton mixing matrix. We adopt the standard parametrization of $U$ as a $3 \times 3$ complex "rotation" matrix, in terms of three mixing angles, $\theta_{12}, \theta_{23}$, and $\theta_{13}$, and one phase, $\delta_{\mathrm{CP}}$. Since neutrinos travel over cosmological distances much longer than the oscillation length, we are only sensitive to the average $v_{\alpha} \rightarrow v_{\beta}$ flavor-transition probability, i.e.,

$$
P_{\alpha \beta}^{\mathrm{std}}=\sum_{i=1}^{3}\left|U_{\alpha i}\right|^{2}\left|U_{\beta i}\right|^{2} .
$$


For present day sentivity, we use and build on the recent NuFit 5.0 global fit [11], which uses data from 31 different analyses of solar, atmospheric, reactor, and accelerator neutrino experiments. We also consider the future measurement of $\sin ^{2} \theta_{12}$ by JUNO, and of $\sin ^{2} \theta_{23}$ and $\delta_{\mathrm{CP}}$ by HK and DUNE. We assume there will be no improvement on $\sin ^{2} \theta_{13}$ presently determined by the Daya Bay experiment. Below we describe the oscillation experiments that we use in our predictions.

JUNO, the Jiangmen Underground Neutrino Observatory [2], will be a 20-kt liquid scintillator detector, located in Guangdong, China. It will measure the oscillation probability $P\left(\bar{v}_{e} \rightarrow \bar{v}_{e}\right)$ of 2-8-MeV reactor neutrinos at a baseline of $\simeq 53 \mathrm{~km}$. JUNO seeks to determine the neutrino mass ordering and precisely measure $\sin ^{2} \theta_{12}$ and $\Delta m_{21}^{2}$. Its nominal sensitivity on $\sin ^{2} \theta_{12}$ is $0.54 \%$ after 6 years of data-taking [2], which is the value we adopt in this work. JUNO is under construction and will start taking data in 2022.

DUNE, the Deep Underground Neutrino Experiment [3], is a long-baseline neutrino oscillation experiment made out of large liquid argon time projection chambers. It will measure the appearance and disappearance probabilities, $P\left(v_{\mu} \rightarrow v_{e}\right)$ and $P\left(v_{\mu} \rightarrow v_{\mu}\right)$, in the $0.5-5-\mathrm{GeV}$ range using accelerator neutrinos, in neutrino and antineutrino modes. DUNE seeks to determine the mass ordering and measure $\delta_{\mathrm{CP}}$ and $\sin ^{2} \theta_{23}$ precisely. We use the official analysis framework released with the DUNE Conceptual Design Report, which is comparable to the DUNE Technical Design Report.

HK is the multipurpose water Cherenkov successor to Super-K, with a fiducial mass of $187 \mathrm{kt}$, under construction in Kamioka, Japan. This long-baseline experiment will measure the appearance and disappearance probabilities of accelerator neutrinos [4]. It operates at slightly lower energies $(\simeq 0.6 \mathrm{GeV})$ and with a shorter baseline $(295 \mathrm{~km})$ than DUNE. Like DUNE, HK will also measure $\delta_{\mathrm{CP}}$ and $\sin ^{2} \theta_{23}$ precisely. It will start operation in 2027 with a projected nominal exposure of 10 years using one Cherenkov tank as far detector.

The left panel of Figure 1 summarizes our projected evolution of the measurement precision of the mixing parameters using a combination of next-generation terrestrial neutrino experiments. Beyond the mixing parameters, we examine how robust are our results to oscillations being non-unitary. The lower panel shows the global limits on non-unitarity from current and future experiments by quantifying the deviation from the normalization $N_{\alpha} \equiv \sum_{i=1}^{3}\left|U_{\alpha i}\right|^{2}=1$, for the $\alpha=e, \mu, \tau$ rows. The sensitivity of the IceCube-Upgrade [5] to $v_{\tau}$ appearance will play a major role in constraining $\sum\left|U_{\tau i}\right|^{2}$.

\section{Future neutrino telescopes}

Below we list the neutrino telescopes we use in our analysis.

IceCube-Gen2 is the planned extension of IceCube [7]. It will add 120 new strings to the existing experiment, leading to an instrumented volume of $7.9 \mathrm{~km}^{3}$ and an effective area that varies from 7 to 8.5 times that of IceCube between $100 \mathrm{TeV}$ and $1 \mathrm{PeV}$. Deployment of IceCube-Gen2 array is planned to start in 2027, with completion expected in 2033. As with IceCube, data taking during the detector construction phase should provide results well before the array is fully operational. Rather than considering the gradual ramp-up of operations between 2027 and 2033, we use an effective start date for the full array of 2030. 

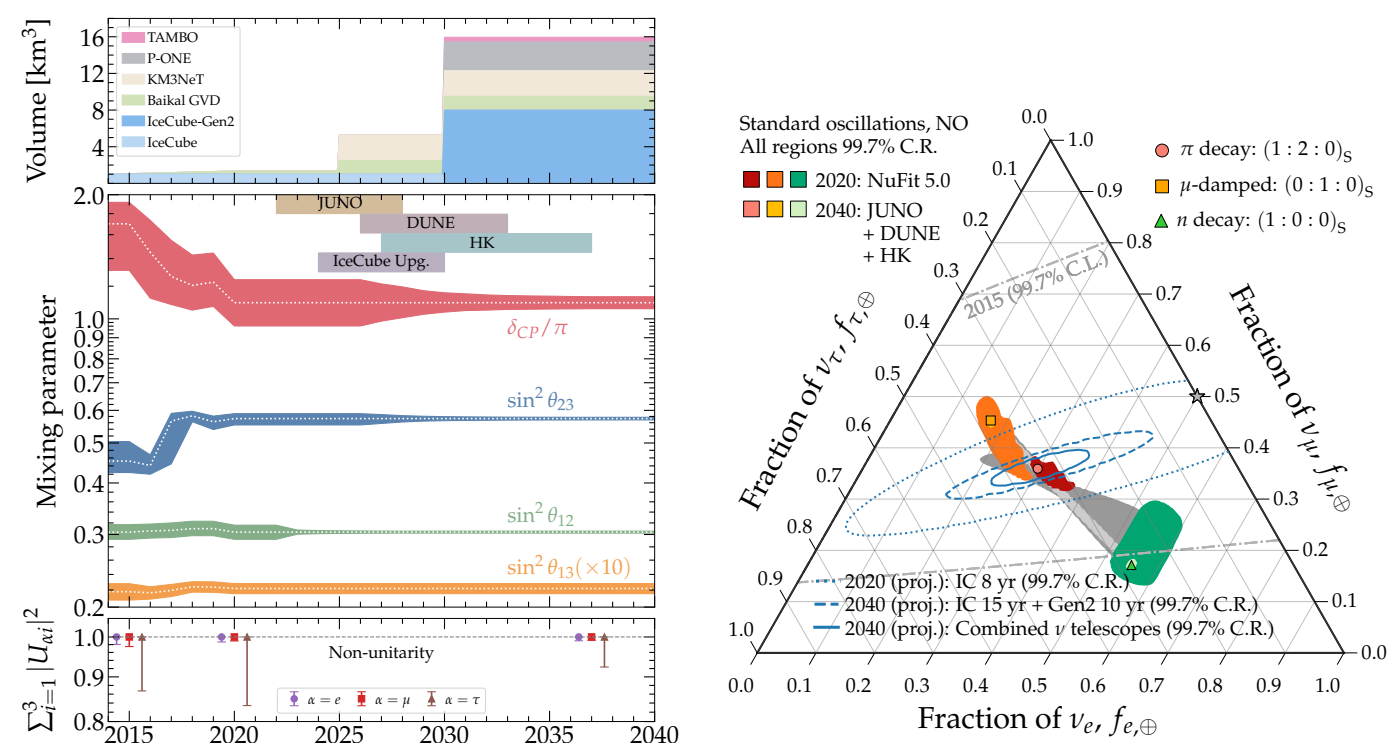

Figure 1: Left panel: The future of neutrino telescopes, oscillation experiments, and flavor mixing measurements. Top: Effective volume of future neutrino telescopes able to probe the flavor composition of astrophysical neutrinos. Center: Time evolution of the oscillation parameters. For each parameter, the dotted white line shows the best-fit value and the shaded region around it, the $1 \sigma$ uncertainty. The boxes at the top show the start and projected estimated running times for these experiments. Bottom: Time evolution of the expected error on the unitarity of the neutrino flavor mixing matrix; values taken from Refs. [12, 13]. Right panel: Comparison between the status of allowed regions of flavor composition at Earth in 2020 and 2040 assuming standard oscillations. Colored regions are computed separately for the three benchmark flavor compositions at the source $\left(f_{e}: f_{\mu}: f_{\tau}\right)_{\mathrm{S}}$ (pion decay, muon damping, neutron decay), and varying over all possible flavor compositions at the source (gray-shaded regions). Lines show the $99.7 \%$ credibility regions (C.R.) of the astrophysical flux assuming a composition of $(0.3,0.36,0.34)_{\oplus}$ at Earth. The overlaid contours show the 2015 IceCube measurement of the flavor composition [14] and projections for IceCube and IceCube-Gen2 [7] and for the combination of all TeV-PeV neutrino telescopes available in 2040, derived here.

KM3NeT [8] is the successor to ANTARES located in the Mediterranean Sea. The high-energy component, called KM3Net/ARCA, will be deployed as two 115-string arrays with 18 DOMs each, $100 \mathrm{~km}$ off the coast of Sicily, and should be complete by 2024. Based on a projected event rate of 15.6 cosmic neutrino-induced cascades per year, we estimate the exposure of KM3Net to be $\simeq 2.4$ times that of IceCube.

Baikal-GVD [6] is a gigaton volume detector that expands on the existing NT-200 detector in lake Baikal, Siberia. The first modules are already installed, and the detector has been operating since 2018 with an effective volume of $0.35 \mathrm{~km}^{3}$. This will rise to $1.5 \mathrm{~km}^{3}$ in 2025 when the detector is complete, consisting of 90 strings, with 12 DOMs each. Baikal-GVD has already seen at least one candidate neutrino cascade event with reconstructed energy of $91 \mathrm{TeV}$.

P-ONE [9], the Pacific Ocean Neutrino Experiment, is a planned water Cherenkov experiment, to be deployed in the Cascadia basin off Vancouver Island, using Ocean Networks Canada infrastructure that is already in place. P-ONE is expected to be complete in 2030 and will include 70 strings, with 20 DOMs each, deployed in a modular array covering a cylindrical volume with a 
$1 \mathrm{~km}$ height and $1 \mathrm{~km}$ radius.

TAMBO [15] the Tau Air-Shower Mountain-Based Observatory, is a proposed array of waterCherenkov tanks to be located in a deep canyon in Peru. TAMBO will search for Earth-skimming $v_{\tau}$ in the $1-100 \mathrm{PeV}$ range. It is expected to detect approximately $7 v_{\tau}$ per year in the energy range considered here. Because it is sensitive to a single flavor, TAMBO will be particularly helpful in breaking the $v_{e}-v_{\tau}$ degeneracy in measuring flavor composition.

We show in Figures 1 right the 99.7\% C.R. 15-year IceCube plus 10-year IceCube-Gen2 contour from Ref. [7], along with the projection of the sensitivities from the combination of future neutrino telescopes by 2040 .

\section{Results}

\subsection{Allowed regions of flavor composition at Earth}

The right panel of Figure 1 shows the 99.7\% C.R. of flavor composition at Earth for the years 2020 and 2040 inferred from our current and future knowledge of neutrino oscillation parameters. The larger gray regions are sampled from a flat prior in source composition, while each of the colored regions assumes the benchmark scenarios: $100 \%$ pion decay (red), muon-damped $\pi$ decay (orange), or neutron decay (green). The reduction in the size of the allowed flavor regions from 2020 to 2040 stems mainly from the improved measurement of $\sin ^{2} \theta_{12}$ by JUNO, which shrinks the regions along the $f_{e, \oplus}$ direction, and of $\sin ^{2} \theta_{23}$ by DUNE and HK, which shrinks the regions along the $f_{\tau, \oplus}$ direction. We have also computed the flavor regions assuming the neutrino mixing is non-unitary, which have approximately the same size as standard oscillations. This means that our ability to pinpoint the dominant mechanism of neutrino production is not affected by the existence of additional neutrino mass states.

\subsection{Inferring the flavor composition at the sources}

Ultimately, we are interested in learning about the identity of the sources of high-energy neutrinos and the physical conditions that govern them. For that we infer the posterior of the electron neutrino fraction at the source $f_{e, S}$ using information from neutrino flavor measurements and neutrino oscillation parameters. We assume that $v_{\tau}$ are not produced in the sources and show the results in the left panel of Figure 2. We find that by 2040 we should be able to recover the true value of $f_{e, S}$ to within $2 \%$ at $68 \%$ C.R..

It is also conceivable that the diffuse flux of high-energy astrophysical neutrinos is due to more than one population of sources and that each population generates neutrinos with a different flavor composition. The right panel of Fig. 2 shows that, while the "true" value of $k_{\pi}=1$ is within the favored region, lower values of $k_{\pi}$ are also allowed, with the same significance, at the cost of increasing the contribution of muon-damped and neutron-decay production. Remarkably, the contribution of neutron-decay production cannot be larger than $40 \%$.

\subsection{Testing new physics}

In order to estimate bounds on the neutrino lifetime, we assume that $v_{2}$ and $v_{3}$ decay invisibly with the same lifetime-to-mass ratio $\tau / m$ and $v_{1}$ is stable. We calculate the diffuse flux of highenergy neutrinos produced by a nondescript population of extragalactic sources, including the effect 

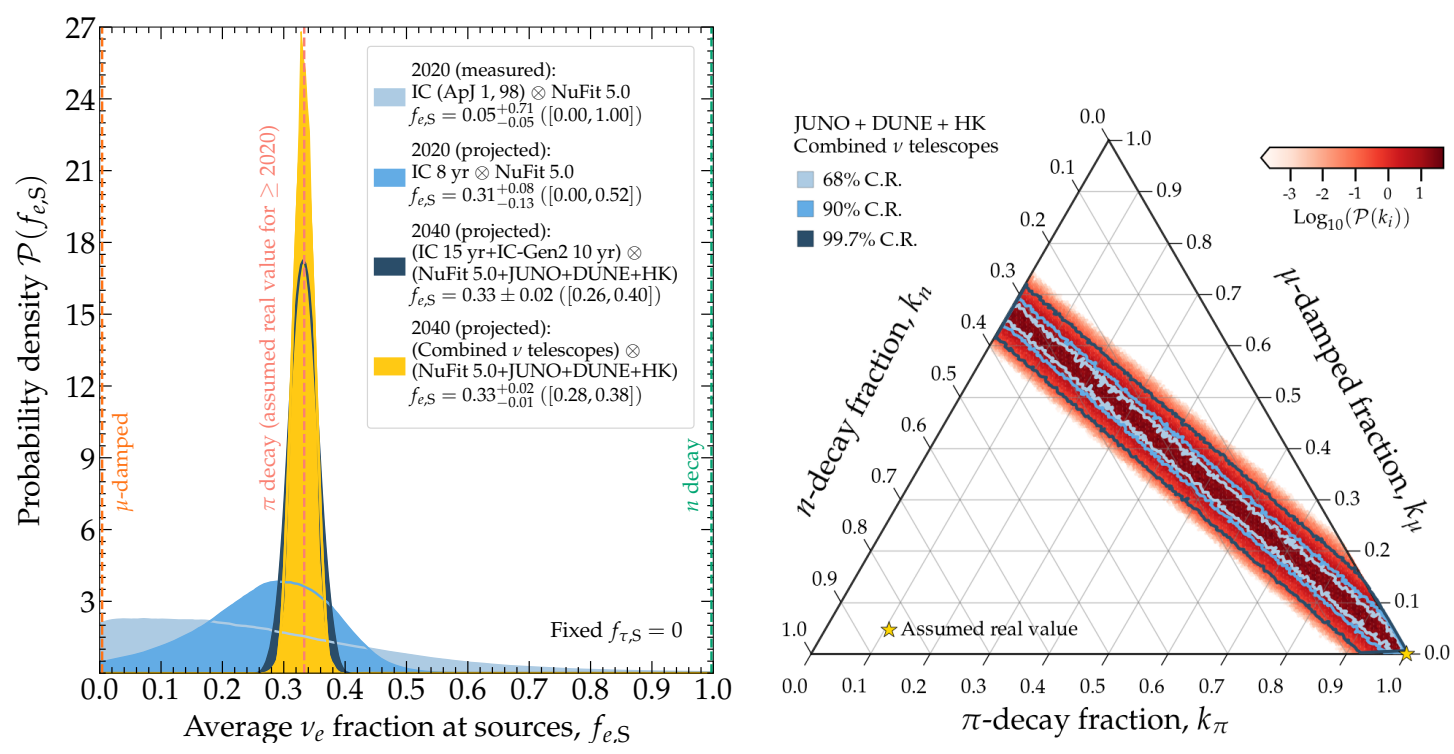

Figure 2: Left: Fraction of $v_{e}$ produced in astrophysical sources, inferred from the flavor composition measured at Earth, in IceCube (IC), IceCube-Gen2 (IC-Gen2), and future neutrino telescopes combined, and accounting for the uncertainties in the mixing parameters. In each case, we show the best-fit value of $f_{e, \mathrm{~S}}$, its $68 \%$ C.R. interval and, in parentheses, its $99.7 \%$ C.R. interval. For the 2020 and 2040 curves, we assume that the real value of $f_{e, \mathrm{~S}}=1 / 3$, coming from the full pion decay chain. We also assume that sources do not produce $v_{\tau}$. Right: Sensitivity to the fraction of the diffuse flux of high-energy neutrinos that is contributed by the three benchmark scenarios. The real value is assumed to be $k_{\pi}=1$, i.e., production only via full pion decay.

of neutrino decay during propagation, following Ref. [16]. Under decay, the flavor composition changes with neutrino energy. In our analysis, we compute the average flavor composition at Earth over the energy interval from $E_{\min }=60 \mathrm{TeV}$ to $E_{\max }=10 \mathrm{PeV}$, and infer the posterior distributions of $m / \tau$ computed assuming that the flavor composition at the source is $\boldsymbol{f}_{\mathrm{S}}=\boldsymbol{f}_{\mathrm{S}}^{\pi} \equiv\left(\frac{1}{3}: \frac{2}{3}: 0\right)_{\mathrm{S}}$. The results are shown in Figure 3. The posteriors translate into lower limits on the lifetimes of $\tau / m \geq 2.4 \times 10^{3}(\mathrm{eV} / m) \mathrm{s}$, using 2015 data, to $5.6 \times 10^{5}(\mathrm{eV} / \mathrm{m}) \mathrm{s}$ in 2040 .

\section{Conclusions}

The flavor composition of high astrophysical neutrinos, has long been regarded as a versatile tool to learn about high-energy astrophysics and test fundamental physics. We have explored the physics potential of neutrino oscillation experiments and neutrino telescopes over the next 20 years. We find that, by 2040, improved measurements of $\theta_{12}$ by JUNO and of $\theta_{23}$ by DUNE and Hyper-Kamiokandewill reduce the size of the allowed flavor regions at Earth predicted by standard oscillations by a factor of 5-10 compared to today, and the combined measurements from BaikalGVD, KM3NeT/ARCA, P-ONE and IceCube-Gen2are expected to reduce the uncertainty in flavor composition by a factor of 2 from 2020 to 2040. Moreover, if high-energy neutrinos are produced by a variety of production mechanisms, each yielding a different flavor composition, we will be able to identify the dominant and sub-dominant mechanisms. To illustrate the improvement that we will achieve in testing beyond-the-Standard-Model neutrino physics using the flavor composition, 

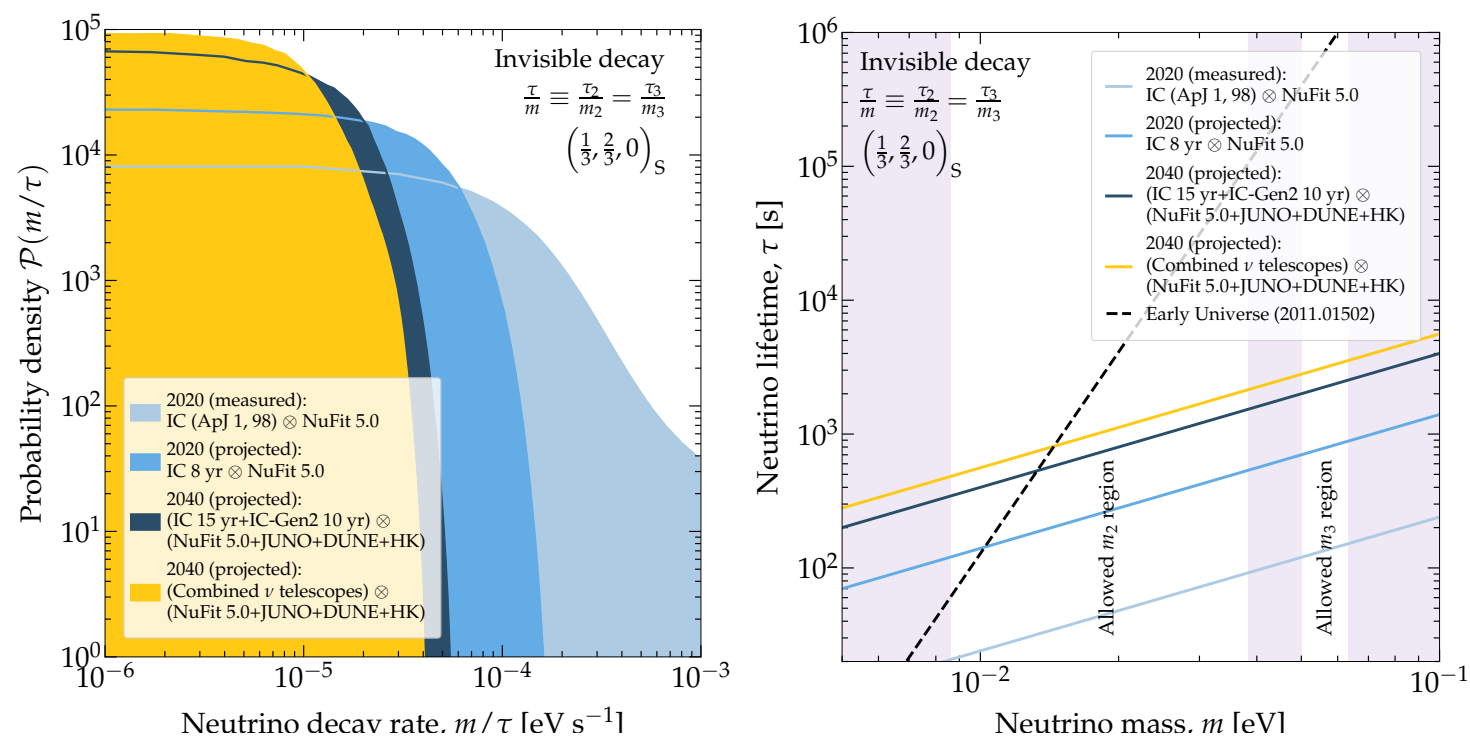

Figure 3: Left: Posterior probability density of the neutrino lifetime $\tau / m \equiv \tau_{2} / m_{2}=\tau_{3} / m_{3}$, extracted assuming invisible decay, and a fixed flavor composition at the source of $\left(\frac{1}{3}, \frac{2}{3}, 0\right)_{S}$. Right: Comparison of the estimated $95 \%$ C.R. lower limits on the lifetime derived here. Low masses are excluded by the measurement of $\Delta m_{i 1}^{2} \equiv m_{i}^{2}-m_{1}^{2}(i=2,3)$ in oscillation experiments [11]; high masses, by cosmological limits on the sum of neutrino masses [17], where we have assumed normal ordering. The early-Universe limit of $\tau>4 \times 10^{5} \mathrm{~s}(\mathrm{~m} / 50 \mathrm{meV})^{5}[18]$ is from CMB and LSS constraints.

we explore neutrino decay into invisible products. We have shown that future observations will be able to constrain the lifetime of the heavier neutrinos to nearly $\sim 10^{5}(\mathrm{eV} / \mathrm{m}) \mathrm{s}$ if only $v_{1}$ is stable.

\section{References}

[1] N. Song, S.W. Li, C.A. Argüelles, M. Bustamante and A.C. Vincent, The Future of High-Energy Astrophysical Neutrino Flavor Measurements, JCAP 04 (2021) 054 [2012.12893].

[2] JUNO collaboration, Neutrino Physics with JUNO, J. Phys. G 43 (2016) 030401 [1507.05613].

[3] DUNE collaboration, Deep Underground Neutrino Experiment (DUNE), Far Detector Technical Design Report, Volume I Introduction to DUNE, JINST 15 (2020) T08008 [2002.02967].

[4] HyPer-KAmioKAnde collaboration, Hyper-Kamiokande Design Report, 1805.04163.

[5] IcECubE collaboration, The IceCube Upgrade - Design and Science Goals, PoS ICRC2019 (2020) 1031 [1908.09441].

[6] BAIKAL-GVD collaboration, The Baikal-GVD neutrino telescope: First results of multi-messenger study, PoS ICRC2019 (2020) 1013 [1908 . 05450]. 
[7] ICECube Gen2 collaboration, IceCube-Gen2: The Window to the Extreme Universe, 2008.04323.

[8] KM3NET collaboration, Letter of intent for KM3NeT 2.0, J. Phys. G 43 (2016) 084001 [1601.07459].

[9] P-ONE collaboration, The Pacific Ocean Neutrino Experiment, Nature Astron. 4 (2020) 913 [2005.09493].

[10] A. Romero-Wolf et al., An Andean Deep-Valley Detector for High-Energy Tau Neutrinos, in Latin American Strategy Forum for Research Infrastructure, 2, 2020 [2002 . 06475].

[11] I. Esteban, M. González-García, M. Maltoni, T. Schwetz and A. Zhou, The fate of hints: updated global analysis of three-flavor neutrino oscillations, JHEP 09 (2020) 178 [2007.14792].

[12] S. Parke and M. Ross-Lonergan, Unitarity and the three flavor neutrino mixing matrix, Phys. Rev. D 93 (2016) 113009 [1508.05095].

[13] S.A.R. Ellis, K.J. Kelly and S.W. Li, Current and Future Neutrino Oscillation Constraints on Leptonic Unitarity, JHEP 12 (2020) 068 [2008. 01088].

[14] ICECUBE collaboration, A combined maximum-likelihood analysis of the high-energy astrophysical neutrino flux measured with IceCube, Astrophys. J. 809 (2015) 98 [1507.03991].

[15] S. Wissel, J. Álvarez-Muñiz, W.R. Carvalho, A. Romero-Wolf, H. Schoorlemmer and E. Zas, A New Concept for High-Elevation Radio Detection of Tau Neutrinos, EPJ Web Conf. 216 (2019) 04007.

[16] M. Bustamante, J.F. Beacom and K. Murase, Testing decay of astrophysical neutrinos with incomplete information, Phys. Rev. D 95 (2017) 063013 [1610.02096].

[17] GAMBIT Cosmology Workgroup collaboration, Strengthening the bound on the mass of the lightest neutrino with terrestrial and cosmological experiments, 2009.03287.

[18] G. Barenboim, J.Z. Chen, S. Hannestad, I.M. Oldengott, T. Tram and Y.Y. Wong, Invisible neutrino decay in precision cosmology, 2011.01502. 